\title{
Dynamical systems analysis of a two level trophic food web in the Southern Oceans
}

\author{
S. A. Hadley ${ }^{1} \quad$ L. K. Forbes ${ }^{2}$
}

(Received 14 February 2008; revised 25 February 2009)

\begin{abstract}
A theoretical model developed by Stone describing a two level trophic system in the Ocean is analyzed, for the case in which there is unlimited supply of nutrients. We show that spontaneous oscillations in population numbers are possible, but they do not arise from a Hopf bifurcation. Seasonal forcing of the model is also investigated, and it is shown that resonances can occur, in addition to highly nonlinear behaviour including high period oscillations, quasi-periodicity and chaos.
\end{abstract}

\section{Contents}

1 Introduction

\section{The mathematical model}

http://anziamj . austms.org.au/ojs/index.php/ANZIAMJ/article/view/1111 gives this article, (c) Austral. Mathematical Soc. 2009. Published March 10, 2009. ISSN 1446-8735. (Print two pages per sheet of paper.) 
3 Analysis of the model E30

3.1 Steady state populations without forcing . . . . . . . . E31

3.2 Stability of steady states ............... E31

4 External forcing

E35

5 Numerical results

E38

6 Discussion

E52

References

E53

\section{Introduction}

Predator-prey interactions have been of great interest both to practical and theoretical biologists, over a long time. In particular, naturally occurring oscillations of populations with time have been studied in a variety of practical situations, and many of these are discussed by Murray [9]. Famous models, such as the Lotka-Volterra system, have been adapted to explain theoretically the source of these oscillations in fish populations, and also in the behaviour of phytoplankton by Edwards and Brindley [2]. Oscillations are possible in the unforced case, for certain choices of parameter values. Thus Huppert et al. [6] found that the rate of zooplankton growth has an effect on blooms in phytoplankton populations. Seasonally related phenomena have been observed in phytoplankton by Freund et al. [3], and this suggests that seasonal forcing of populations can also be an important consideration.

Stone [11] studied a two level trophic web found in the Southern Oceans. He sought, in particular, to explain the paradoxical nature of the interaction between the phytoplankton and bacteria that form part of the system. Both species compete for the same inorganic nutrients, that may be of limited availability. When these nutrients are scarce, the phytoplankton release 
extracellular organic carbon that is used by the bacteria. In essence, the phytoplankton directly promote the survival of a competitor. This is intuitively at odds with the nature of competition.

To explain this phenomenon, Stone [11] referred to the concept of a reductionist approach to the interaction between species, commonly invoked by ecologists. In this viewpoint, behaviour is deduced only from the interactions between organisms in isolation, rather than by treating the many interactions of a community as an integrated system. However, the apparently paradoxical behaviour may have an explanation when the system is instead considered as a whole. For example, if phytoplankton are disadvantaged by the presence of bacteria, which in turn are predated by protozoa, then it is conceivable that the phytoplankton, by stimulating the growth of bacteria, may be also stimulating the growth of the protozoa that graze on them. This may prove advantageous to the phytoplankton. This is an instance of the "Paradox of Enrichment" discussed by Kirk [7], in which adding more prey results in population cycles that increase in amplitude. Gross et al. [4] showed that the dynamics of a general predator-prey system can be either stabilized or destabilized by enhancing prey numbers, depending on the form of the interaction function between species (or "compartments" more generally, if, for example, nutrients are involved as a variable).

This article analyses Stone's [11] two level trophic web model of the interaction between phytoplankton and bacteria, in which protozoa and zooplankton are also included. The purpose here is not to extend or alter Stone's model, which we take as a reliable description of the population behaviour, but rather to explore thoroughly the dynamical behaviour that it predicts. Concepts from the theory of dynamical systems [9, 1, e.g.] determine the stability of steady state populations and the possibility of self sustained oscillations. This type of analysis has not previously been applied to Stone's [11] model, and in this investigation we make the simplifying assumption that nutrients are present in such abundance that they play no role in the overall dynamics. Nevertheless, it is seen that complex behaviour, including the presence of multiple steady states, is possible in this (four variable) model. A simi- 
lar conclusion was reached by Truscott and Brindley [13] in a much simpler (two variable) model of phytoplankton-zooplankton interaction. Their study treated the population dynamics as being equivalent to reactions in an excitable medium, and it allowed for nutrient scarcity, in part, through the use of Michaelis-Menten type grazing functions. They found that multiple stationary states and even limit cycle oscillations were possible in their two variable system, which could predict at least some of the qualitative features of phytoplankton blooms.

Here, we also allow the growth rate of bacteria to vary sinusoidally, as a model for seasonal (or diurnal) fluctuations in the environment. The mathematical model is non-linear and, as a consequence, complicated resonance behaviour is possible in the solution. Furthermore, even the presence of quasi-periodicity and chaos is suggested for certain parameter values.

Section 2 presents the model and, for convenience, scaled (non-dimensional) populations and rates are introduced in a similar fashion to Truscott and Brindley [13]. Section 3 provides an analysis of the basic (unforced) model. Section 4 presents a linearized analysis of the forced model, in which we assume small amplitude for the forcing term. Two (primary) resonance peaks are shown to be present. Numerical solutions to the non-linear forced model equations are presented and discussed in Section 5. Multiple resonance peaks and even possible quasi-periodic and chaotic behaviours are found. A discussion in Section 6 concludes the article.

\section{The mathematical model}

We consider the two level trophic system illustrated in Figure 1, taken from the model proposed by Stone [11]. There are five interacting compartments, namely the Bacteria (B), Phytoplankton (P), Zooplankton (Z), Protozoa (R) and Nutrients $(\mathrm{N})$, and these are indicated in the diagram. The arrows show positive interaction between components in the model. We consider the case 


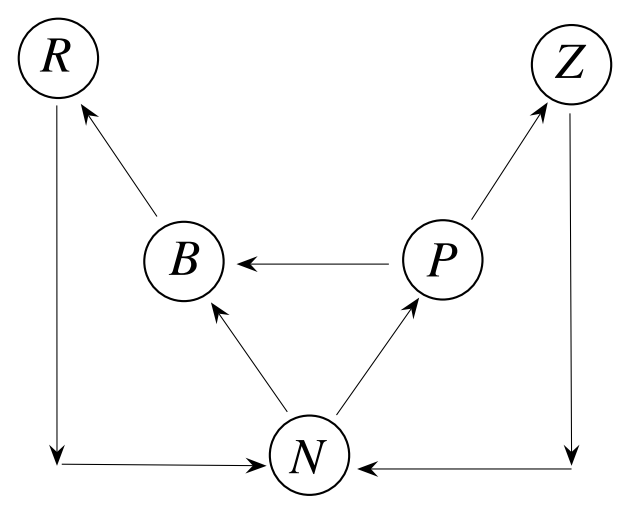

Figure 1: Stone's Compartmental model showing interaction between species: R, B, N, P, Z represent protozoa, bacteria, nutrients, phytoplankton, zooplankton, respectively. The direction of the arrows indicates a direct positive influence by one component on another. The effect of nutrients $\mathrm{N}$ is ignored in the present study.

in which the nutrient concentration $\mathrm{N}$ is inexhaustible, and therefore not subject to change. The interactions in the Figure 1 then give rise to the system of four ordinary differential equations

$$
\begin{aligned}
\mathrm{dB} / \mathrm{dt} & =r_{\mathrm{b}} \mathrm{B}-\mathrm{r}_{\mathrm{r}} \mathrm{RB}+\mathrm{r}_{i} \mathrm{~PB}, \\
\mathrm{dP} / \mathrm{dt} & =r_{\mathrm{p}} \mathrm{P}-\mathrm{r}_{z} \mathrm{PZ}-\mathrm{r}_{i} \mathrm{~PB}, \\
\mathrm{~d} Z / \mathrm{dt} & =r_{z} \mathrm{PZ}-\mathrm{d}_{z} \mathrm{Z}, \\
\mathrm{d} R / \mathrm{dt} & =r_{r} \mathrm{RB}-\mathrm{d}_{\mathrm{r}} \mathrm{R},
\end{aligned}
$$

for the time dependent behaviour of the populations of the four species. In Stone's original model [11], the interaction between bacteria and phytoplankton was described as an example of commensalism, in which bacteria $\mathrm{B}$ benefitted from phytoplankton P, but without cost to P. However, here we assume a simpler situation that interactions between any two compartments illustrated in Figure 1 result in gain to one and loss to the other.

In this system (1), the symbol $r_{b}$ denotes the growth rate of the bacteria, 
and $r_{p}$ is the reproduction rate for phytoplankton. The three quantities $r_{r}, r_{i}$ and $r_{z}$ are the (second order) interaction rates between protozoa and bacteria, phytoplankton and bacteria and phytoplankton and zooplankton, respectively. The remaining factors $d_{r}$ and $d_{z}$ are the mortality rates of protozoa and zooplankton. All these are positive quantities.

We undertake an analysis of the dynamical behaviour of the system (1) in the unforced case in which all the parameters are constants. Of particular interest are conditions necessary for a solution to exhibit oscillatory behaviour. If possible, we also wish to identify those situations in which a limit cycle might be born by means of a Hopf bifurcation, which occurs when a non-linear selfsustained oscillation appears directly from a steady state population as a parameter is varied [9].

We are also interested in the effect of subjecting the system (1) to external periodic forcing, arising physically from seasonal or daily variations in the environment. This is achieved mathematically by representing the reproduction rate for bacteria $r_{b}$ in the form

$$
r_{b}(t)=r_{b 0}+r_{b 1} \cos (\omega t) .
$$

Here, $r_{b 0}$ is the average breeding rate and $r_{b 1}$ is its forcing amplitude. The constant $\omega$ is the frequency of the seasonal forcing, and has units day ${ }^{-1}$. As indicated by Edwards and Brindley [2], this term indicates that bacteria are more likely to reproduce in daylight.

The original system of equations (1) is now re-cast in terms of dimensionless variables. This simplifies the model as it results in fewer dimensionless groupings of parameters, rather than isolated model parameters that must be varied individually in order to analyse the model fully. All four populations $(B, P, Z, R)$ are scaled with respect to the quantity $r_{p} / r_{r}$ which is a naturally occurring measure of population size implicit in equations (1). Similarly, time $t$ is made dimensionless using $1 / r_{p}$, which is a time scale linked roughly to the lifecycle of the phytoplankton. In these non-dimensional vari- 
ables, equations (1) become

$$
\begin{aligned}
& \mathrm{dB} / \mathrm{dt}=\beta \mathrm{B}-\mathrm{RB}+\eta \mathrm{PB}, \\
& \mathrm{dP} / \mathrm{dt}=\mathrm{P}-\alpha \mathrm{PZ}-\eta \mathrm{PB}, \\
& \mathrm{dZ} / \mathrm{dt}=\alpha \mathrm{PZ}-\delta \mathrm{Z}, \\
& \mathrm{dR} / \mathrm{dt}=\mathrm{RB}-\gamma \mathrm{R} .
\end{aligned}
$$

There are thus four dimensionless parameter groupings in the system (3):

$$
\alpha=r_{z} / r_{r}, \quad \gamma=d_{r} / r_{p}, \quad \delta=d_{z} / r_{p}, \quad \eta=r_{i} / r_{r} .
$$

The first of these parameters, $\alpha$, represents the reproduction rate of phytoplankton. The second, $\gamma$, corresponds to the mortality rate of the protozoa. The third parameter, $\delta$, is the mortality rate of the zooplankton and the fourth quantity, $\eta$, is the relative rate of interaction between the bacteria and phytoplankton. All four parameters are constants, and in addition there is a time dependent reproduction rate for bacteria

$$
\beta(t)=\beta_{0}+\beta_{1} \cos (\Omega t),
$$

from equation (2). Equation (5) introduces three additional non-dimensional parameters

$$
\beta_{0}=r_{b 0} / r_{p}, \quad \beta_{1}=r_{b 1} / r_{p}, \quad \Omega=\omega / r_{p} .
$$

The first of these is the steady state reproduction rate for bacteria. The second parameter, $\beta_{1}$, is the seasonal forcing amplitude for that reproduction rate, and the final parameter, $\Omega$, is its forcing frequency relative to the time scale for natural bacterial growth. Thus the model is fully described by the set of seven constants in equations (4) and (6).

\section{Analysis of the model}

This section begins by considering the dynamics of the system (3) without seasonal forcing, so that $\beta_{1}=0$. 


\subsection{Steady state populations without forcing}

Steady states are solutions (B, P, Z, R) which satisfy

$$
\mathrm{dB} / \mathrm{dt}=\mathrm{dP} / \mathrm{dt}=\mathrm{dZ} / \mathrm{dt}=\mathrm{dR} / \mathrm{dt}=0 .
$$

There are five separate equilibria for the non-dimensional model system (3):

$$
\begin{aligned}
& \left(B_{\text {eq }}, P_{\text {eq }}, Z_{\text {eq }}, R_{\text {eq }}\right)=(0,0,0,0) \\
& \left(B_{\text {eq }}, P_{\text {eq }}, Z_{\text {eq }}, R_{\text {eq }}\right)=\left(1 / \eta,-\beta_{0} / \eta, 0,0\right), \\
& \left(B_{\text {eq }}, P_{\text {eq }}, Z_{\text {eq }}, R_{\text {eq }}\right)=\left(\gamma, 0,0, \beta_{0}\right) \\
& \left(B_{\text {eq }}, P_{\text {eq }}, Z_{\text {eq }}, R_{\text {eq }}\right)=(0, \delta / \alpha, 1 / \alpha, 0), \\
& \left(B_{\text {eq }}, P_{\text {eq }}, Z_{\text {eq }}, R_{\text {eq }}\right)=\left(\gamma, \delta / \alpha,(1-\gamma \delta) / \alpha, \beta_{0}+\eta \delta / \alpha\right) .
\end{aligned}
$$

The first steady state in equation (7) represents the case where all four species become extinct. In the second steady state, two of the species survive but the population of one of these is negative and thus not physically meaningful. For the third and fourth steady states, two of the species survive and two again become extinct. The surviving species in these two states form an independent predator-prey system, each involving two species only, and correspond closely to the famous Lotka-Volterra system [9]. The fifth and final steady state in (7) is potentially of most interest here, as it represents the situation when all four species survive.

\subsection{Stability of steady states}

When the time dependent populations are close to any of the five steady states in equations (7), the small amplitude behaviour is determined by linearization, in the form

$$
\begin{aligned}
& \mathrm{B}(\mathrm{t})=\mathrm{B}_{\text {eq }}+\epsilon \mathrm{B}_{1}(\mathrm{t})+\mathcal{O}\left(\epsilon^{2}\right), \\
& \mathrm{P}(\mathrm{t})=\mathrm{P}_{\text {eq }}+\epsilon \mathrm{P}_{1}(\mathrm{t})+\mathcal{O}\left(\epsilon^{2}\right),
\end{aligned}
$$




$$
\begin{aligned}
& Z(t)=Z_{\text {eq }}+\epsilon Z_{1}(t)+\mathcal{O}\left(\epsilon^{2}\right), \\
& R(t)=R_{\text {eq }}+\epsilon R_{1}(t)+\mathcal{O}\left(\epsilon^{2}\right) .
\end{aligned}
$$

The constant $\epsilon$ is supposed to be small, and represents a measure of how close the system is to one of its steady states (7). The linearized system near an equilibrium point is determined by substituting these forms (8) into the governing equations (3) and retaining only terms of first order in $\epsilon$. This results in the linear matrix system

$$
\frac{d}{d t}\left[\begin{array}{l}
B_{1} \\
P_{1} \\
Z_{1} \\
R_{1}
\end{array}\right]=\left[\begin{array}{clrr}
J_{11} & \eta B_{e q} & 0 & -B_{e q} \\
-\eta P_{e q} & J_{22} & -\alpha P_{e q} & 0 \\
0 & \alpha Z_{e q} & -\alpha \delta P_{\text {eq }} & 0 \\
R_{e q} & 0 & 0 & J_{44}
\end{array}\right]\left[\begin{array}{l}
B_{1} \\
P_{1} \\
Z_{1} \\
R_{1}
\end{array}\right]
$$

where, for convenience, we define intermediate quantities

$$
\begin{aligned}
& \mathrm{J}_{11}=\beta_{0}-\mathrm{R}_{\mathrm{eq}}+\eta \mathrm{P}_{\mathrm{eq}}, \\
& \mathrm{J}_{22}=1-\alpha \mathrm{Z}_{\mathrm{eq}}-\eta \mathrm{B}_{\mathrm{eq}}, \\
& \mathrm{J}_{44}=\mathrm{B}_{\mathrm{eq}}-\gamma .
\end{aligned}
$$

We substitute, in turn, each equilibrium point into equation (9) to determine the eigenvalues of the $4 \times 4$ constant (Jacobian) coefficient matrix for that steady state. In each case, the four eigenvalues so obtained determine the behaviour of the linearized system near the corresponding equilibrium point.

From this analysis it is determined that the first point $(0,0,0,0)$ in $(7)$ is a saddle, with eigenvalues $\beta_{0}, 1,-\delta$ and $\gamma$. Similarly, the second point $\left(1 / \eta,-\beta_{0} / \eta, 0,0\right)$ is also a saddle, and its eigenvalues are $1 / \eta-\gamma,-\alpha \beta_{0} / \eta-\delta$ and $\pm \beta_{0}$. The third point $\left(\gamma, 0,0, \beta_{0}\right)$ in $(7)$ has eigenvalues $1-\eta \gamma,-\delta$, $\pm i \sqrt{\beta_{0} \gamma}$, and the point $(0, \delta / \alpha, 1 / \alpha, 0)$ has eigenvalues $\beta_{0}+\eta \delta / \alpha,-\gamma$, $\pm i \sqrt{\delta}$. Both these points are essentially saddles, in the sense that they combine stable behaviour (negative eigenvalues) with unstable (positive eigenvalues). However, they both possess a pair of purely imaginary eigenvalues, and this gives them an oscillatory behaviour in some plane passing through 
the equilibrium point in the phase space. In this regard these two equilibria could give similar oscillatory behaviour to the famous Lotka-Volterra system, in which a non-linear centre occurs [9].

The steady state of most practical interest is the fifth point $(\gamma, \delta / \alpha,(1-$ $\left.\gamma \delta) / \alpha, \beta_{0}+\eta \delta / \alpha\right)$ in the system (7), at which none of the populations disappear. The eigenvalues $\lambda$ for this case are found from the equation

$$
\operatorname{det}\left[\begin{array}{clrr}
-\lambda & \eta \gamma & 0 & -\gamma \\
-\eta \delta / \alpha & -\lambda & -\delta & 0 \\
0 & 1-\eta \gamma & -\lambda & 0 \\
\beta_{0}+\eta \delta / \alpha & 0 & 0 & -\lambda
\end{array}\right]=0 .
$$

This is expanded to give the quartic equation

$$
\lambda^{4}+T \lambda^{2}+D=0
$$

in which it is convenient to define the quantities

$$
\begin{aligned}
\mathrm{T} & =\gamma\left(\beta_{0}+\eta \delta / \alpha\right)+\delta(1-\eta \gamma)+\eta^{2} \gamma \delta / \alpha, \\
\mathrm{D} & =\gamma \delta(1-\eta \gamma)\left(\beta_{0}+\eta \delta / \alpha\right) .
\end{aligned}
$$

The quartic equation (10) for the eigenvalues $\lambda$ has the solution

$$
\lambda^{2}=\left[-\mathrm{T} \pm \sqrt{\mathrm{T}^{2}-4 \mathrm{D}}\right] / 2 .
$$

There are four eigenvalues $\lambda$ as solutions to (12). If any two of them form a complex conjugate pair that crosses the imaginary axis as a parameter is varied, a Hopf bifurcation is possible [5, p. 151]. Thus a necessary condition for limit cycle generation in this way is that the real part of a complex eigenvalue pair changes sign. It is convenient to introduce the notation

$$
X=\beta_{0}+\eta \delta / \alpha, \quad Y=1-\eta \gamma, \quad S=\eta^{2} \gamma \delta / \alpha,
$$

in terms of which the quantities in equation (11) take the simpler forms

$$
\mathrm{T}=\gamma \mathrm{X}+\delta \mathrm{Y}+\mathrm{S}, \quad \mathrm{D}=\gamma \delta \mathrm{XY} .
$$


We observe that $\mathrm{D}>0$ in equations (11) and (14), since the parameter values given in Stone's [11] article show that $1-\eta \gamma>0$. For a Hopf bifurcation to occur, the real part of $\lambda$ in equation (12) must change sign, and vanish at a particular parameter value (the Hopf bifurcation value), for non-zero imaginary part. A necessary condition for this to occur is therefore that

$$
\mathrm{T}>0 \text { and } \mathrm{T}^{2}-4 \mathrm{D}>0 .
$$

However, it follows from equations (13) and (14) that

$$
\mathrm{T}^{2}-4 \mathrm{D}=\gamma^{2} \mathrm{X}^{2}+2 \gamma \mathrm{X}(\mathrm{S}-\delta \mathrm{Y})+(\mathrm{S}+\delta \mathrm{Y})^{2} .
$$

The right-hand side of equation (16) is an irreducibly positive quadratic in $X$, for all $Y>0$. Thus the inequalities in (15) are satisfied for all $Y>0$, so that the real part of $\lambda$ in equation (12) cannot change sign, but is always zero. This shows that there is no Hopf bifurcation in the system (3) for $\eta \gamma<1$, which is the case of practical interest as indicated by Stone [11].

Although the system has no Hopf bifurcation, it follows from (15) and (12) that the eigenvalues for this steady state are

$$
\lambda= \pm i \sqrt{\left[\mathrm{T} \pm \sqrt{\left.\mathrm{T}^{2}-4 \mathrm{D}\right]} / 2\right.}
$$

This means that the linearized system predicts a neutrally stable centre at this equilibrium, surrounded by concentric periodic orbits [8, p. 40]. However, this is not necessarily an indication of the behaviour of the corresponding non-linear system (3), since the Hartman linearization theorem fails at a centre $[5$, p. 13 , e.g.], and additional information is needed in order to establish the behaviour of the non-linear system near the equilibrium. Accordingly, we examined the full system (3) numerically and observed non-linear centre behaviour in the unforced system. 


\section{External forcing}

Due to seasonal or diurnal influences, it is now assumed that the growth rate $\beta$ for bacteria is not constant, but varies with time as detailed in equation (5). We now consider that the seasonal forcing amplitude $\beta_{1}$ in (5) is a small parameter, and linearize about the fifth steady state point in equation (7) using perturbation expansions of the form

$$
\begin{aligned}
& \mathrm{B}(\mathrm{t})=\mathrm{B}_{\text {eq }}+\beta_{1} \mathrm{~B}_{1}(\mathrm{t})+\mathcal{O}\left(\beta_{1}^{2}\right), \\
& \mathrm{P}(\mathrm{t})=\mathrm{P}_{\text {eq }}+\beta_{1} \mathrm{P}_{1}(\mathrm{t})+\mathcal{O}\left(\beta_{1}^{2}\right), \\
& \mathrm{Z}(\mathrm{t})=\mathrm{Z}_{\text {eq }}+\beta_{1} \mathrm{Z}_{1}(\mathrm{t})+\mathcal{O}\left(\beta_{1}^{2}\right), \\
& \mathrm{R}(\mathrm{t})=\mathrm{R}_{\text {eq }}+\beta_{1} \mathrm{R}_{1}(\mathrm{t})+\mathcal{O}\left(\beta_{1}^{2}\right) .
\end{aligned}
$$

When equations (18) are substituted into the governing system (3) and (5), for the fifth equilibrium in (7), and terms are retained to first order in $\beta_{1}$, there results the linear system of forced equations

$$
\begin{aligned}
\mathrm{dB}_{1} / \mathrm{dt} & =\gamma \cos (\Omega \mathrm{t})-\gamma \mathrm{R}_{1}+\gamma \eta \mathrm{P}_{1}, \\
\mathrm{dP}_{1} / \mathrm{dt} & =-\delta \mathrm{Z}_{1}-(\eta \delta / \alpha) \mathrm{B}_{1}, \\
\mathrm{dZ}_{1} / \mathrm{dt} & =\mathrm{YP}_{1}, \\
\mathrm{dR}_{1} / \mathrm{dt} & =\mathrm{XB}_{1},
\end{aligned}
$$

in which the two constants $\mathrm{X}$ and $\mathrm{Y}$ are as defined in equation (13).

It is known that periodic solutions to (19) are of the form

$$
\begin{aligned}
& B_{1}(t)=a_{1} \cos (\Omega t)+b_{1} \sin (\Omega t), \\
& P_{1}(t)=a_{2} \cos (\Omega t)+b_{2} \sin (\Omega t), \\
& Z_{1}(t)=a_{3} \cos (\Omega t)+b_{3} \sin (\Omega t), \\
& R_{1}(t)=a_{4} \cos (\Omega t)+b_{4} \sin (\Omega t) .
\end{aligned}
$$

After some algebra, the amplitude constants in equations (20) may be shown to be given by the relations

$$
a_{1}=0, \quad b_{1}=-(\Omega L) /(M X),
$$




$$
\begin{array}{ll}
\mathrm{a}_{2}=\Omega^{2} \eta \delta \gamma / M, & \mathrm{~b}_{2}=0, \\
\mathrm{a}_{3}=0, & \mathrm{~b}_{3}=\Omega \bigvee \eta \delta \gamma / M, \\
\mathrm{a}_{4}=\mathrm{L} / \mathrm{M}, & \mathrm{b}_{4}=0,
\end{array}
$$

in which the quantities

$$
\begin{aligned}
L & =-\alpha \gamma X\left(\Omega^{2}-\delta Y\right), \\
M & =\alpha \Omega^{4}-\left(\alpha \gamma X+\alpha \delta Y+\eta^{2} \gamma \delta\right) \Omega^{2}+\alpha \gamma \delta X Y,
\end{aligned}
$$

have been defined for convenience. This linearized solution (20) breaks down whenever the amplitude constants in equations (21) fail to be defined. This is the point of primary resonance in the forced system. It occurs when $M=0$, leading at once to the quartic equation

$$
\Omega^{4}-\mathrm{T} \Omega^{2}+\gamma \delta \mathrm{XY}=0
$$

for the frequency $\Omega$. The quantities $T, X$ and $Y$ are as defined in equations (11) and (13).

It follows from equation (23) that resonance occurs at the frequencies

$$
\Omega=\sqrt{\left[T \pm \sqrt{T^{2}-4 D}\right] / 2} .
$$

Of particular interest here is the relationship between the resonant frequencies given by (24) and the eigenvalues $\lambda$ in equation (17): $\Omega=\sqrt{-\lambda^{2}}$. This shows that resonance occurs precisely when the forcing frequency matches the naturally occurring (centre) oscillations near the equilibrium point.

For the trophic web system discussed by Stone [11], we now evaluate the resonant frequencies (24) explicitly. From parameter values given by Stone, we estimate the dimensionless constants of the present article to have values $\alpha=0.4, \beta_{0}=1.2, \gamma=1.2, \delta=0.4$ and $\eta=0.2$. It follows from equation (11) that $\mathrm{T}=2.0320$ and $\mathrm{D}=0.5107$. These parameter values are assumed hereafter. Equation (24) therefore shows that there are two 


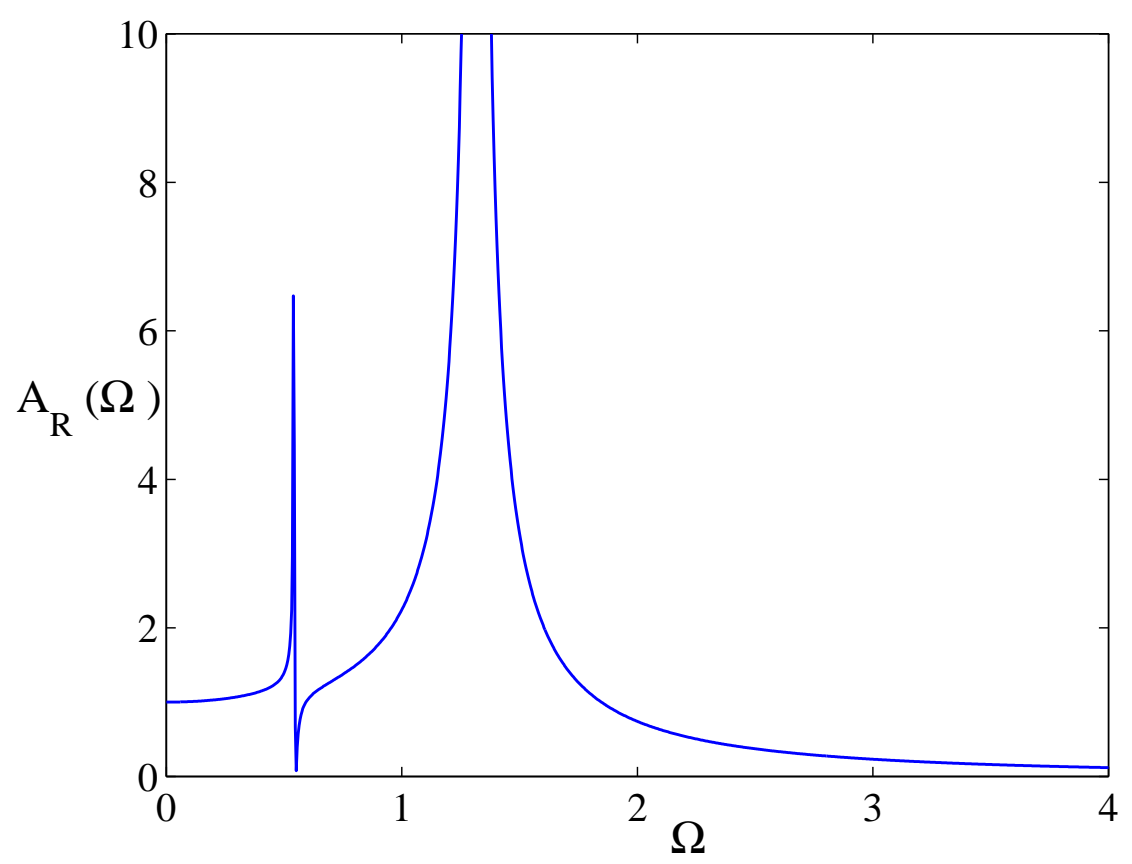

FigURE 2: The resonance curve showing solution amplitude against forcing frequency for the linearized solution $R_{1}(t)$.

frequencies $\Omega=0.5421$ and $\Omega=1.3184$ at which primary resonance occurs. In dimensional variables, these are equivalent to periods of about 15 hours and 7 hours, so that these two primary resonances occur roughly at diurnal forcing frequencies.

Figure 2 depicts the change in amplitude $|L / M|$ of the solution $R_{1}(t)$ of the linear system (19) as forcing frequency $\Omega$ varies. The diagram shows the two points of primary resonance at $\Omega=0.5421$ and $\Omega=1.3184$, where the amplitude of the linearized solution becomes infinite. Of course, linearization itself is only valid for small amplitudes, and so the linearized solution breaks down near resonance, and non-linear effects dominate. The amplitude in 
Figure 2 falls to zero at the forcing frequency $\Omega=\sqrt{\delta \mathrm{Y}}=0.5514$, where $\mathrm{L}=0$ in equation (22).

\section{$5 \quad$ Numerical results}

This section presents the results of numerical solutions to the fully nonlinear system of equations (3) with seasonal forcing term (5). The differential equations were integrated in time using the package MATLAB.

Figures $3-5$ show a sequence of results of the amplitude of the solution $R(t)$ against the forcing frequency $\Omega$, for three different forcing amplitudes $\beta_{1}$. The period of oscillation in the case of seasonal forcing is $\tau=2 \pi / \Omega$.

The results in Figures 3-5 were generated by the following method. A differential equation integration routine from the package MATLAB was used to find the solution to the system of equations (3), subject to the seasonal forcing term (5). The initial point was taken to be $(\gamma, \delta / \alpha,(1-\delta \gamma) / \alpha, X)$, and the dimensionless constants $\alpha$, and so on, were given the values described previously. The numerical solution was integrated forward in time for a large number of forcing periods, typically of the order of $800 \tau$, until transients had died away. The solution $R(t)$ was then recorded for a further 15 forcing periods, and the maximum amplitude for each of these successive periods was plotted as a point on the graph. This process was repeated at each forcing frequency $\Omega$. This permits the effects of resonance and nonlinearity to be examined, since a single point on the diagram at a particular frequency corresponds to a period-one solution at that frequency. Two points represents a period-doubled solution, and so on. A continuum of points at a fixed frequency indicates either quasi-periodicity or chaos. Figures 3-5 effectively give Poincaré cross-sections at each frequency [5, p. 22], and so can be regarded as bifurcation plots.

In Figure 3, a small forcing amplitude $\beta_{1}=0.005$ was used. Two primary resonance peaks are visible in the diagram, at frequencies very close to the 


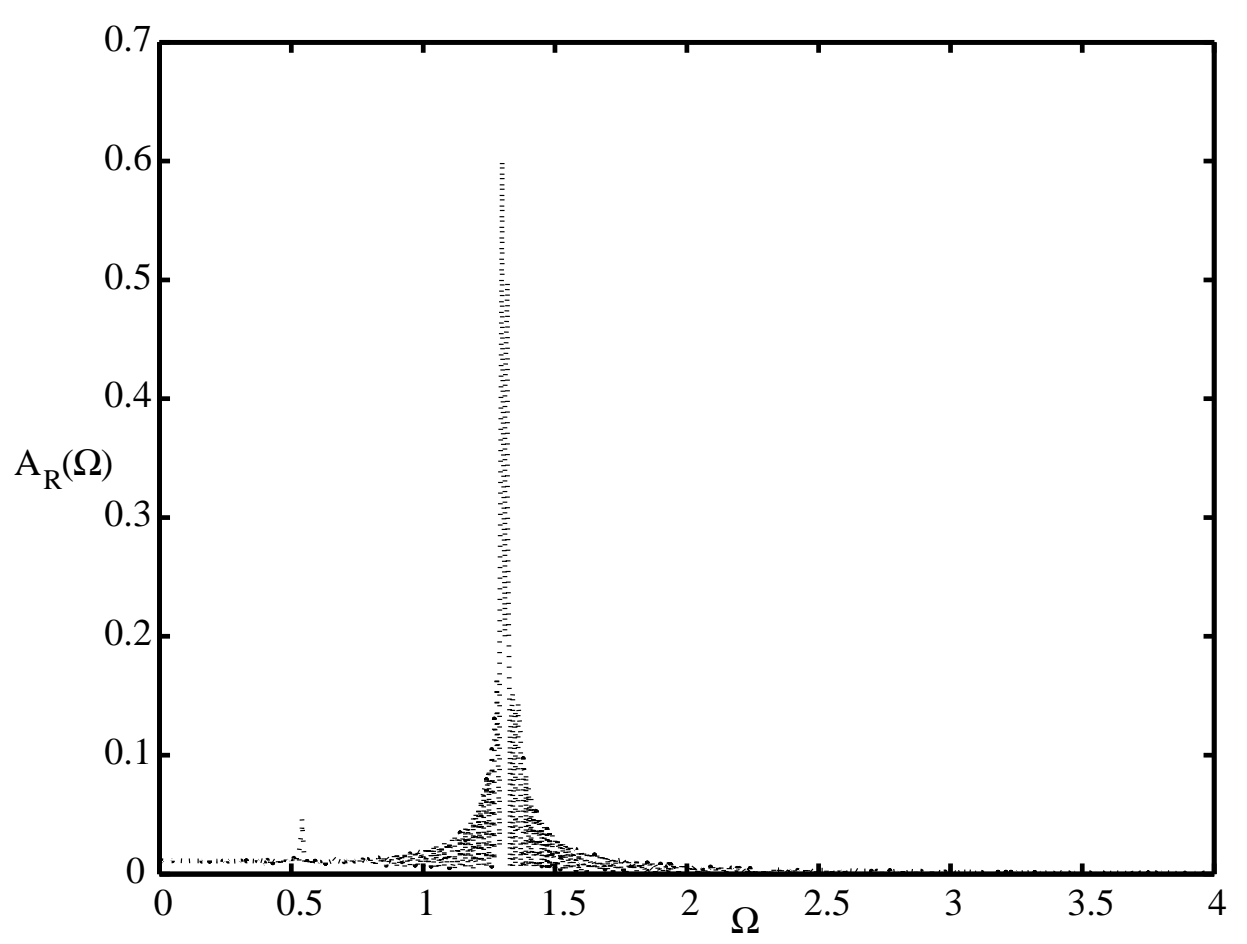

Figure 3: Amplitude of the non linear solution $R(t)$ against forcing frequency $\Omega$, for small forcing amplitude $\beta_{1}=0.005$. 


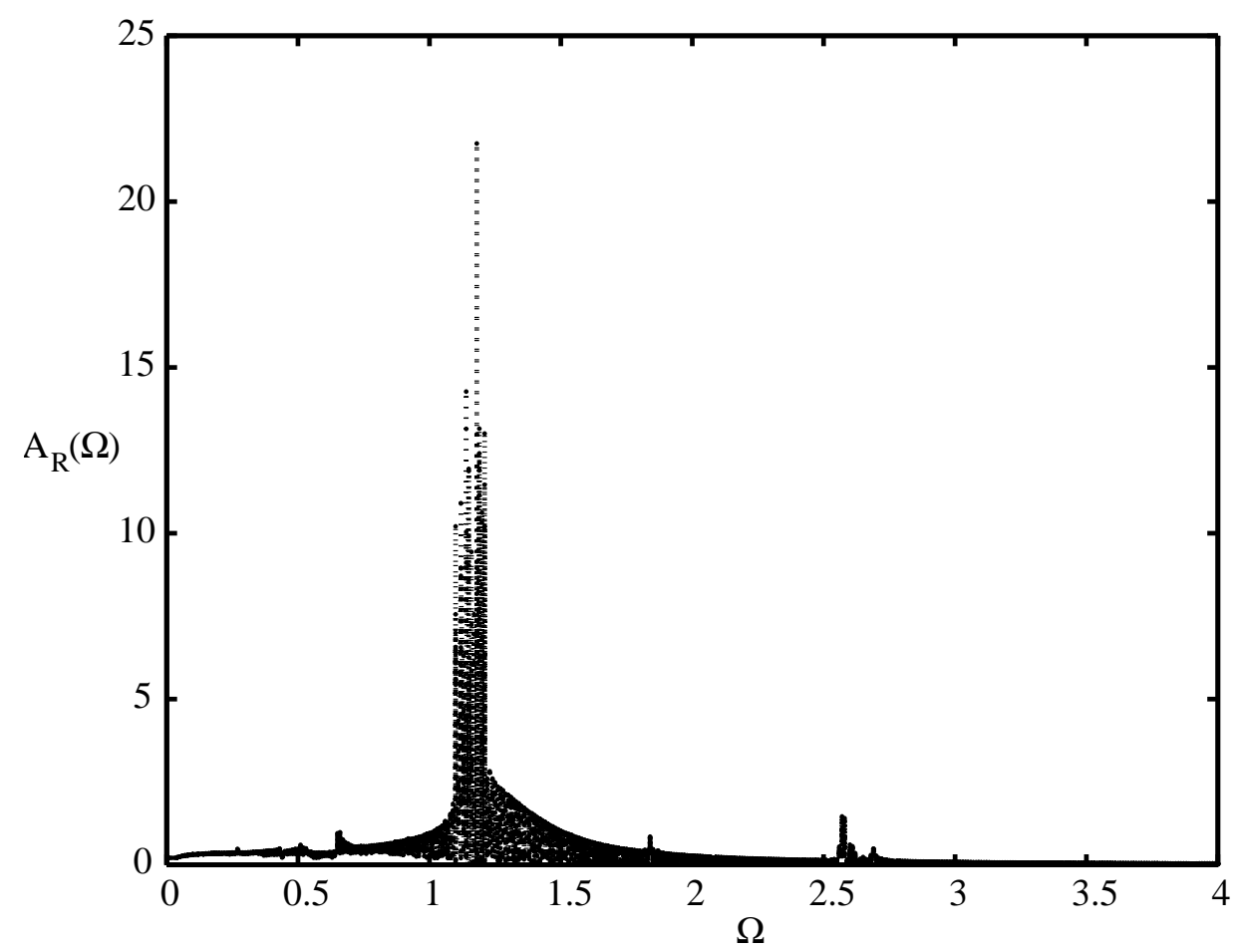

Figure 4: Amplitude of the non linear solution $R(t)$ against forcing frequency $\Omega$, for moderate forcing amplitude $\beta_{1}=0.2$. 


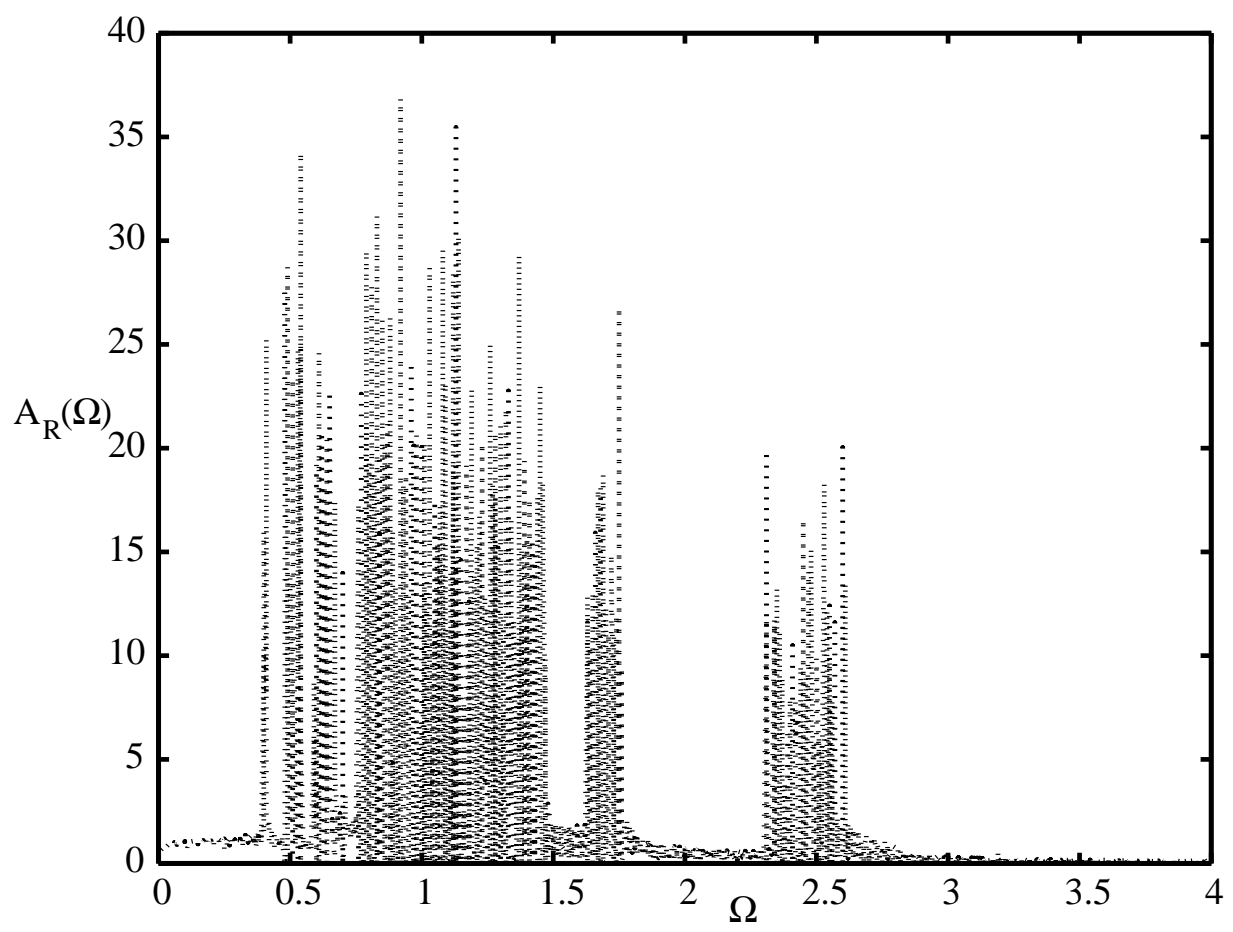

Figure 5: Amplitude of the non linear solution $R(t)$ against forcing frequency $\Omega$, for large forcing amplitude $\beta_{1}=0.6$. 
linearized resonances $\Omega=0.5421$ and $\Omega=1.3184$. For this small forcing amplitude, the non-linear results in Figure 3 resemble closely the linearized solution in Figure 2, as is expected. This confirms the reliability of the present approach.

The forcing amplitude was increased to the moderate value $\beta_{1}=0.2$ to produce the results in Figure 4. It is evident that the agreement with the linearized solution begins to break down at this forcing amplitude. There is no longer a uniform rise to a peak at resonance, and instead the peak has broadened significantly. An additional sub-harmonic resonance peak is also visible at a frequency of about $\Omega \approx 2.5$, and is evidence of the increasing role of non-linearity in this solution.

Figure 5 shows further increases in the effects of non-linearity. This figure was obtained with large forcing amplitude $\beta_{1}=0.6$. The primary resonance peak has now been replaced with a broad band of large amplitude forced responses, apparently containing primary and sub-harmonic resonances along with chaotic responses. A secondary large amplitude peak is visible at about $\Omega \approx 2.5$.

In the next sequence of diagrams, Figures $6-8$, the forcing frequency $\Omega$ is held constant, and the linearized and non-linear solutions for $\mathrm{R}(\mathrm{t})$ are compared for different values of forcing amplitude.

The linearized solution in Figures $6-8$ has been calculated from the result for $R(t)$ given in equation (20), and is sketched with dashed lines in these diagrams. The non-linear solutions were computed using MATLAB to integrate (3) forward in time for 100 periods $(100 \tau)$, to remove transients due to initial conditions, and then a further ten periods were computed and are presented with solid lines in these graphs. (The same initial conditions were assumed as for the bifurcation diagrams in Figures 3-5). The forcing frequency $\Omega=1.362$ was chosen to be close to the upper primary resonance in the linearized solution.

The results in Figure 6 show that the non-linear solution is in reasonably close 


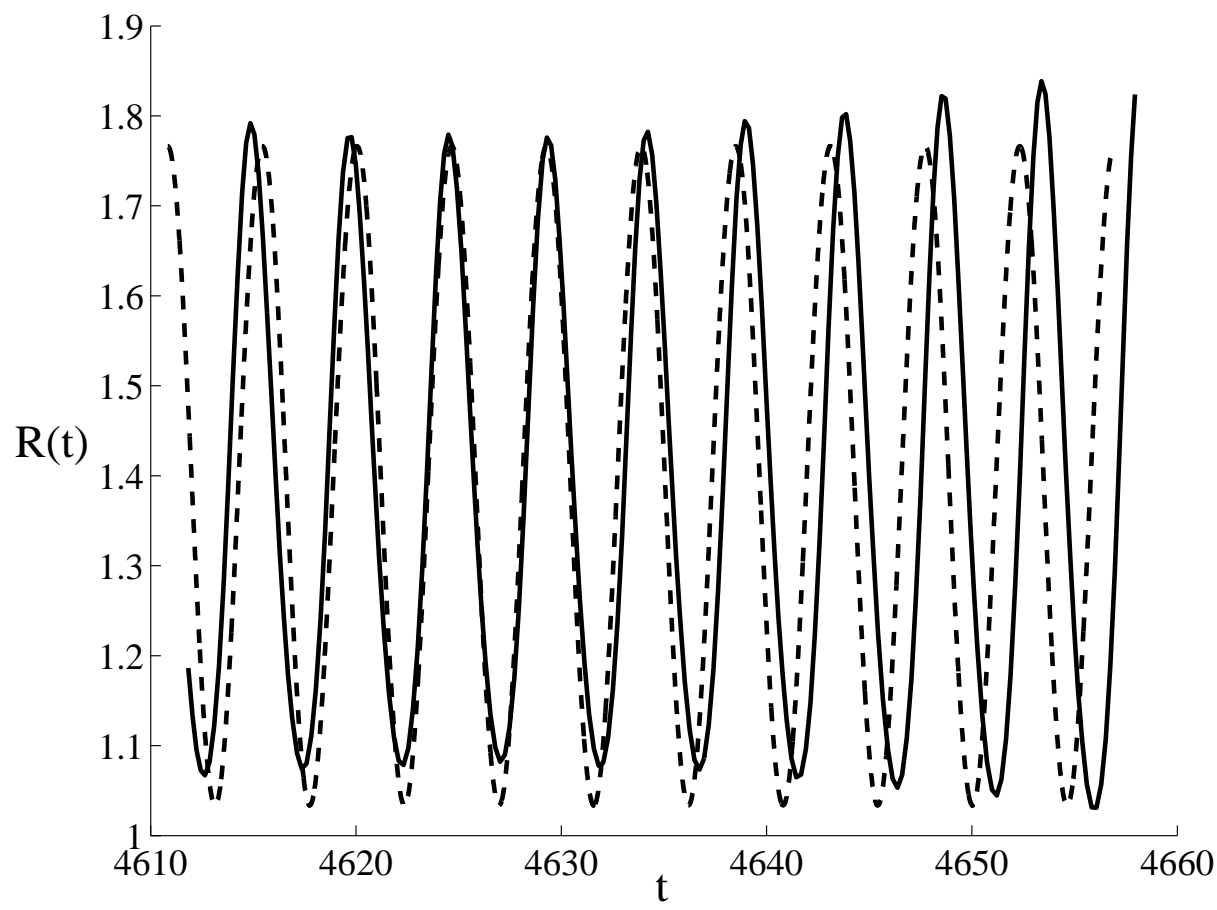

FiguRE 6: Linearized solution (dashed line) compared with the non-linear solution (solid line) for $R(t)$ with amplitude $\beta_{1}=0.005$ and frequency $\Omega=$ 1.362 . 


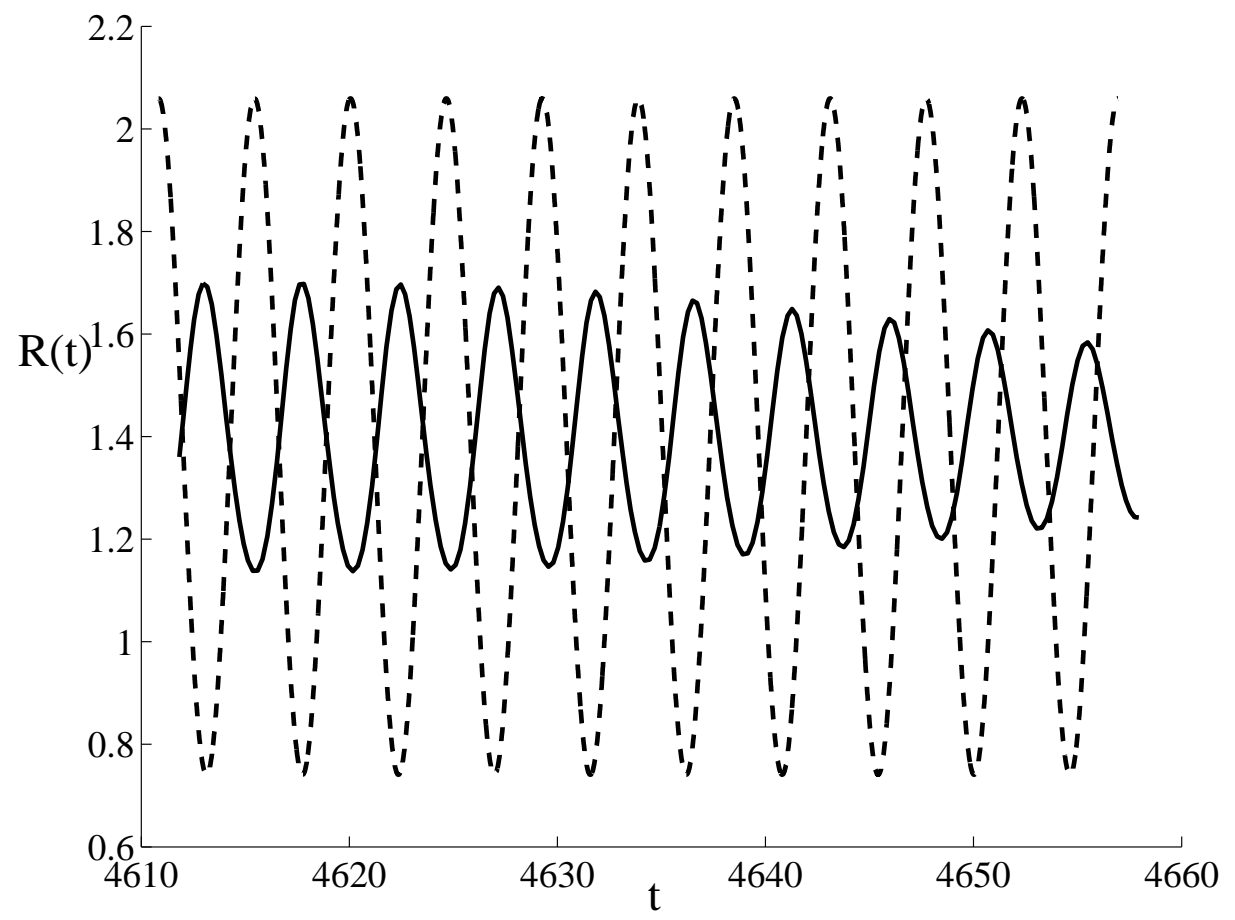

FiguRE 7: Linearized solution (dashed line) compared with the non-linear solution (solid line) for $R(t)$ with amplitude $\beta_{1}=0.009$ and frequency $\Omega=$ 1.362 . 


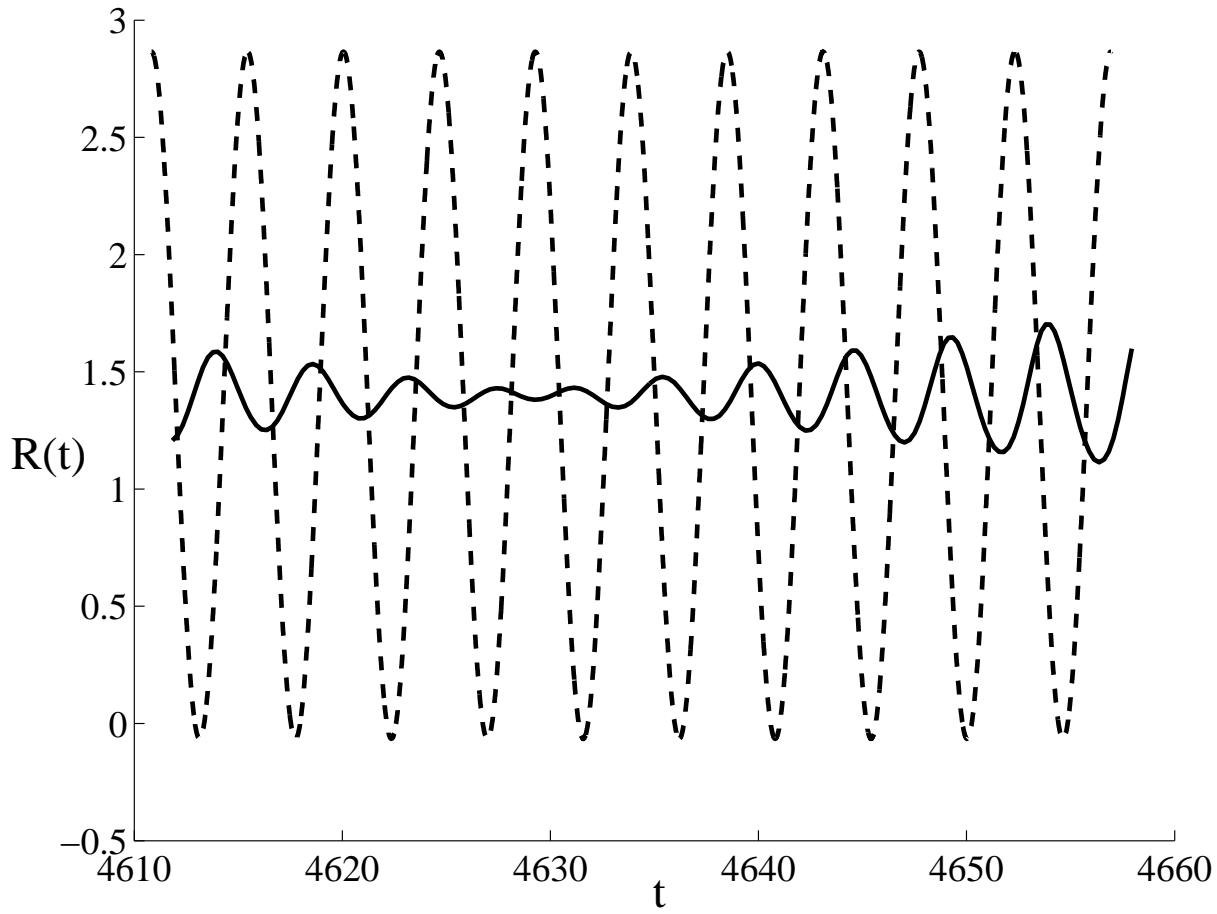

FigURE 8: Linearized solution (dashed line) compared with the non-linear solution (solid line) for $R(t)$ with amplitude $\beta_{1}=0.02$ and frequency $\Omega=$ 1.362 . 
agreement with the non-linear profile shown, as is broadly to be expected for this small forcing amplitude $\beta_{1}=0.005$. This confirms the reliability of the integration routine. Nevertheless, even at this small forcing amplitude, there is evidence of some non-linear effects. In particular, the amplitude of the non-linear solution is not quite constant, suggesting the influence of a high period perturbation, and the period itself is slightly different from that of the purely linearized result.

These effects are more strongly evident in Figure 7. The amplitude of the non-linear solution decreases for the ten forcing periods shown in the diagram, but increases again at later times, giving clear evidence for the existence of a high period orbit. This appears to be associated with quasi-periodic behaviour, as the solution is dominated by several different frequency components that are not rational multiples of one another. This is discussed again later.

The final solution shown in Figure 8 gives further evidence of the high-period behaviour of the non-linear signal. It is interesting to observe that, for this case, the non-linear solution has very much smaller amplitude than its linearized counterpart. Of course, the linearized solution in Figure 8 cannot be expected to retain any validity for this largest forcing amplitude $\beta_{1}$, in particular since the solution in equation (18) predicts that the response amplitude simply increases linearly with forcing amplitude $\beta_{1}$. This would eventually generate negative values for the solution $R(t)$ itself: the result in Figure 8 is the largest value of forcing amplitude $\beta_{1}$ for which the linearized solution remains positive for all time.

In Figures 9 and 10, the non-linear solution is examined further, for forcing amplitudes $\beta_{1}$ larger than those shown in Figures $6-8$. In these next two diagrams, comparison with the linearized solution is no longer possible, as discussed above.

The forcing amplitude in Figure 9 has been increased to $\beta_{1}=0.05$ and 30 periods $(30 \tau)$ have now been shown, to give a clear indication of the behaviour of the solution $R(t)$. Evidently, the response curve has indeed become quasi- 


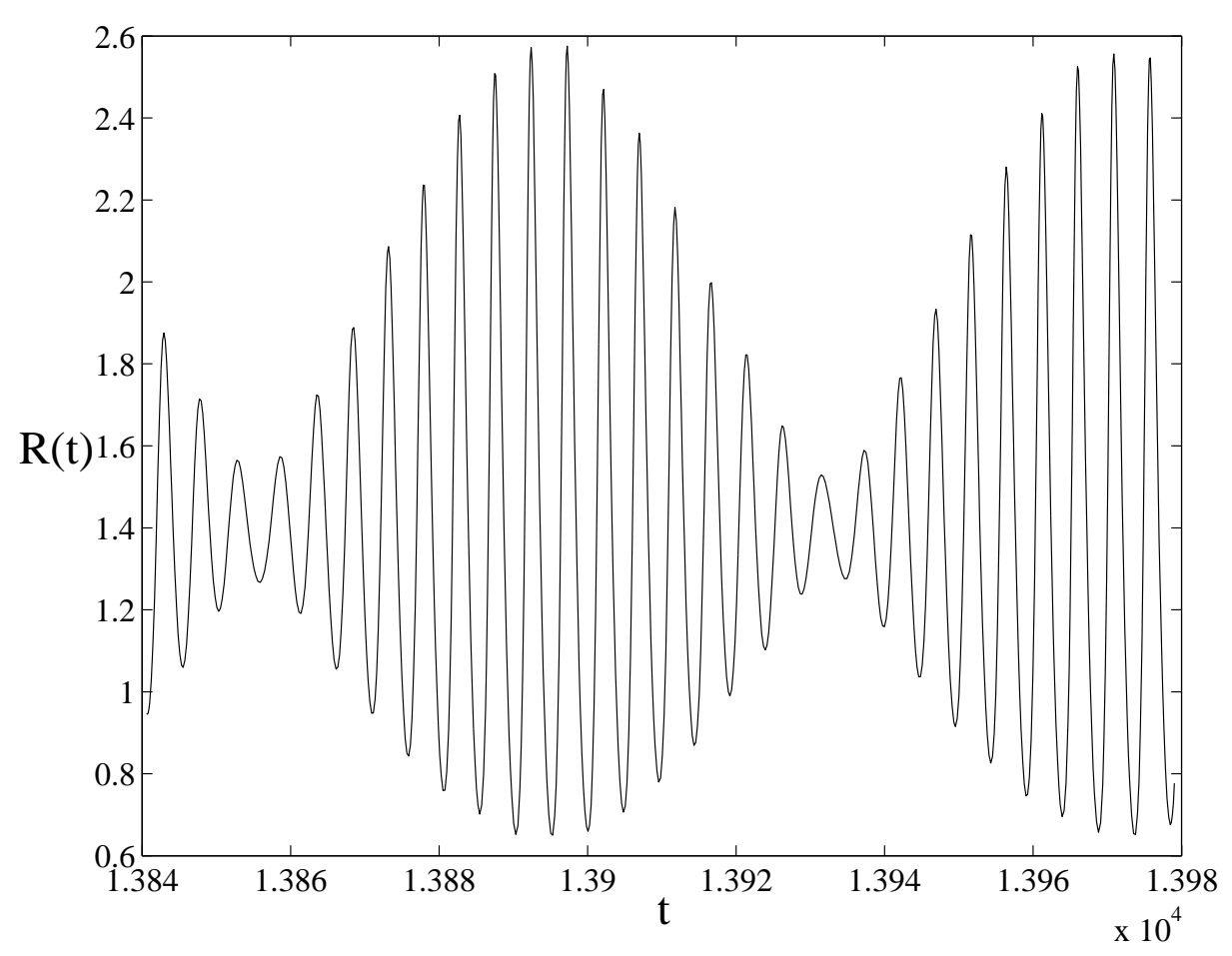

Figure 9: Non-linear solution for $R(t)$ as a function of time, for amplitude $\beta_{1}=0.05$ and frequency $\Omega=1.362$. 


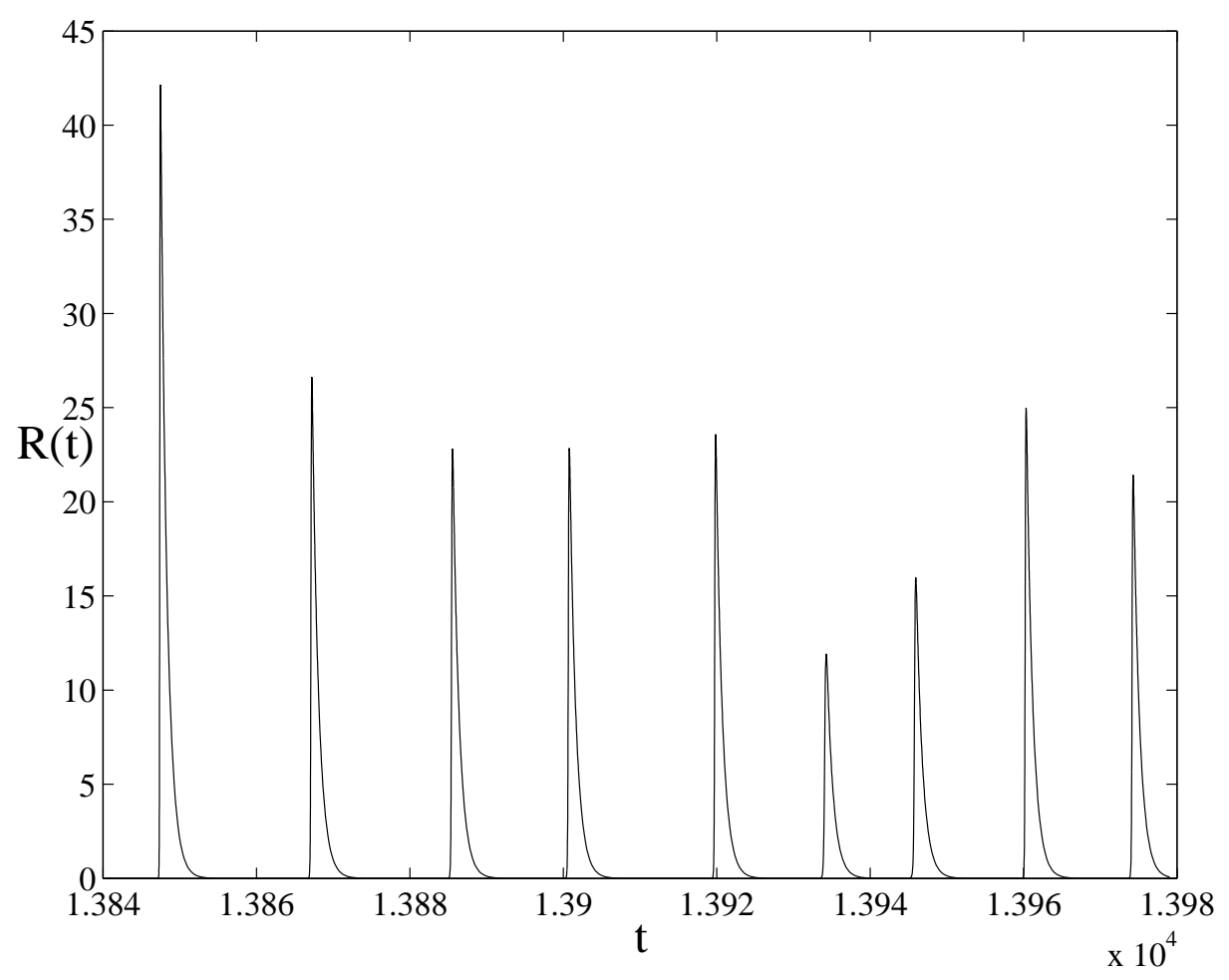

Figure 10: Non-linear solution for $\mathrm{R}(\mathrm{t})$ as a function of time, for amplitude $\beta_{1}=0.2$ and frequency $\Omega=1.362$. 
periodic, with two dominant frequencies. Careful examination of the signal strongly suggests that the solution is not periodic (this is illustrated in Figure 11). Quasi-periodicity is known to be a possible route to chaos, as shown in the theorem of Ruelle, Takens and Newhouse (as discussed by Thompson and Stewart [12, p. 196]). In a recent examination of high dimensional Lotka-Volterra systems, Sprott et al. [10] likewise found that such systems exhibited quasi-periodic behaviour en route to chaos.

For the result in Figure 10, the forcing amplitude was increased further to $\beta_{1}=0.2$. The solution for $R(t)$ now displays intervals of relaxation between large peaks, all of dissimilar amplitudes and occurring apparently at random intervals. This stochastic behaviour of the solution, coupled with its extreme sensitivity to initial conditions, suggests strongly that chaotic motion is generated for this forcing amplitude at this frequency.

Further support for the contention that Figures 9 and 10 exhibit quasiperiodic and chaotic behaviours, respectively, is obtained by considering the solution orbits in the phase space. As the system (3) is actually four dimensional, it is not possible to display the full space, but meaningful results are nevertheless obtained by considering a two dimensional projection onto a plane. This is done in Figures 11 and 12 for the two solutions displayed in Figures 9 and 10, using orbits in a plane consisting of the two variables $R(t)$ and $B(t)$. Physically, these represent the populations of the protozoa and the bacteria, and 30 periods are shown.

Figure 11 shows a region in the (R, B)-plane in which the solution trajectories evidently fill a ring-shaped portion of the plane. This is strongly suggestive of quasi-periodicity, and corresponds to a projection of a high dimensional torus onto the (R, B)-plane. The results in Figure 12 for the larger forcing amplitude $\beta_{1}=0.2$, however, are more strongly suggestive of chaos. There is clearly no longer a torus structure, but instead the solution trajectories appear to move randomly within some bounded region in the space. 


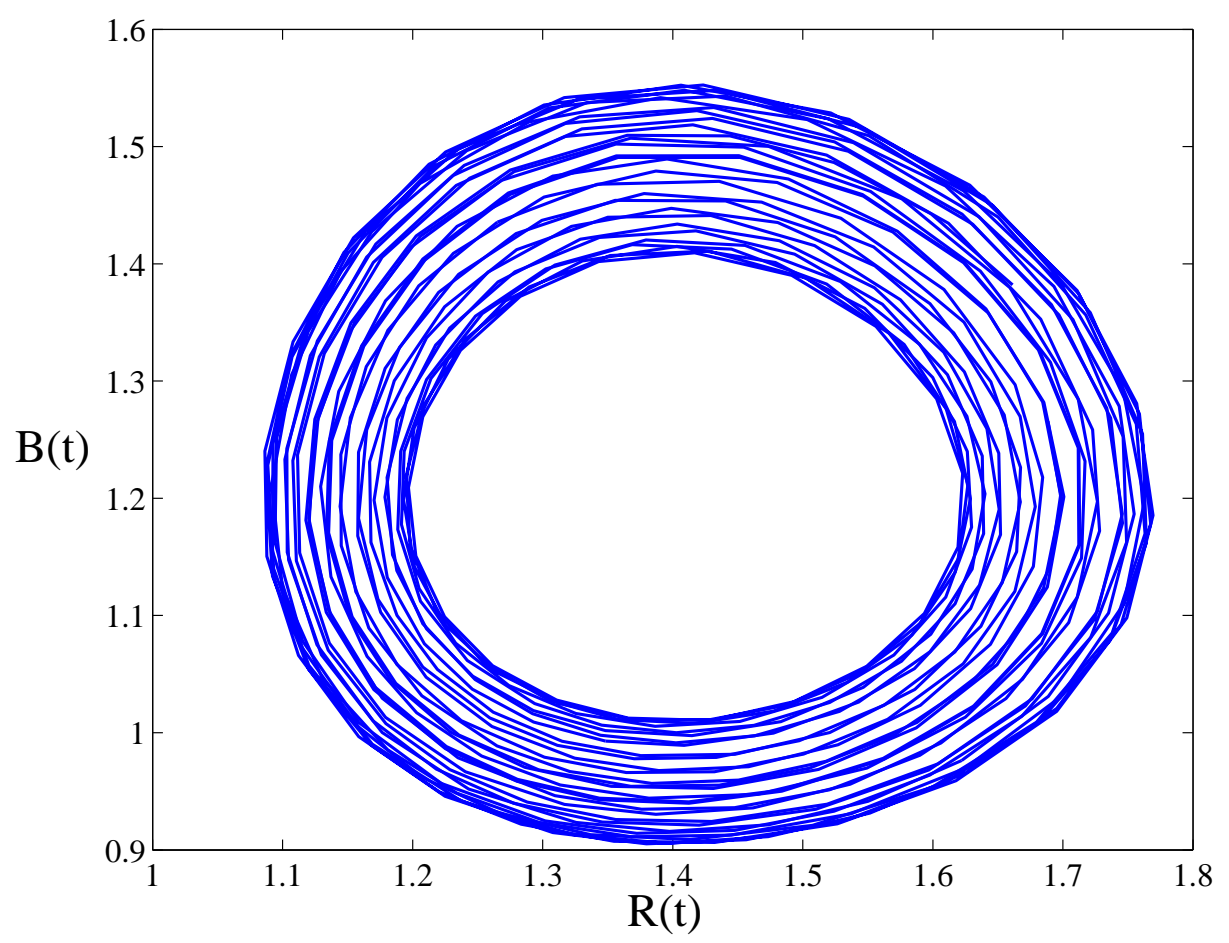

Figure 11: Phase orbits for $B(t)$ against $R(t)$, for forcing amplitude $\beta_{1}=$ 0.05 and frequency $\Omega=1.362$. 


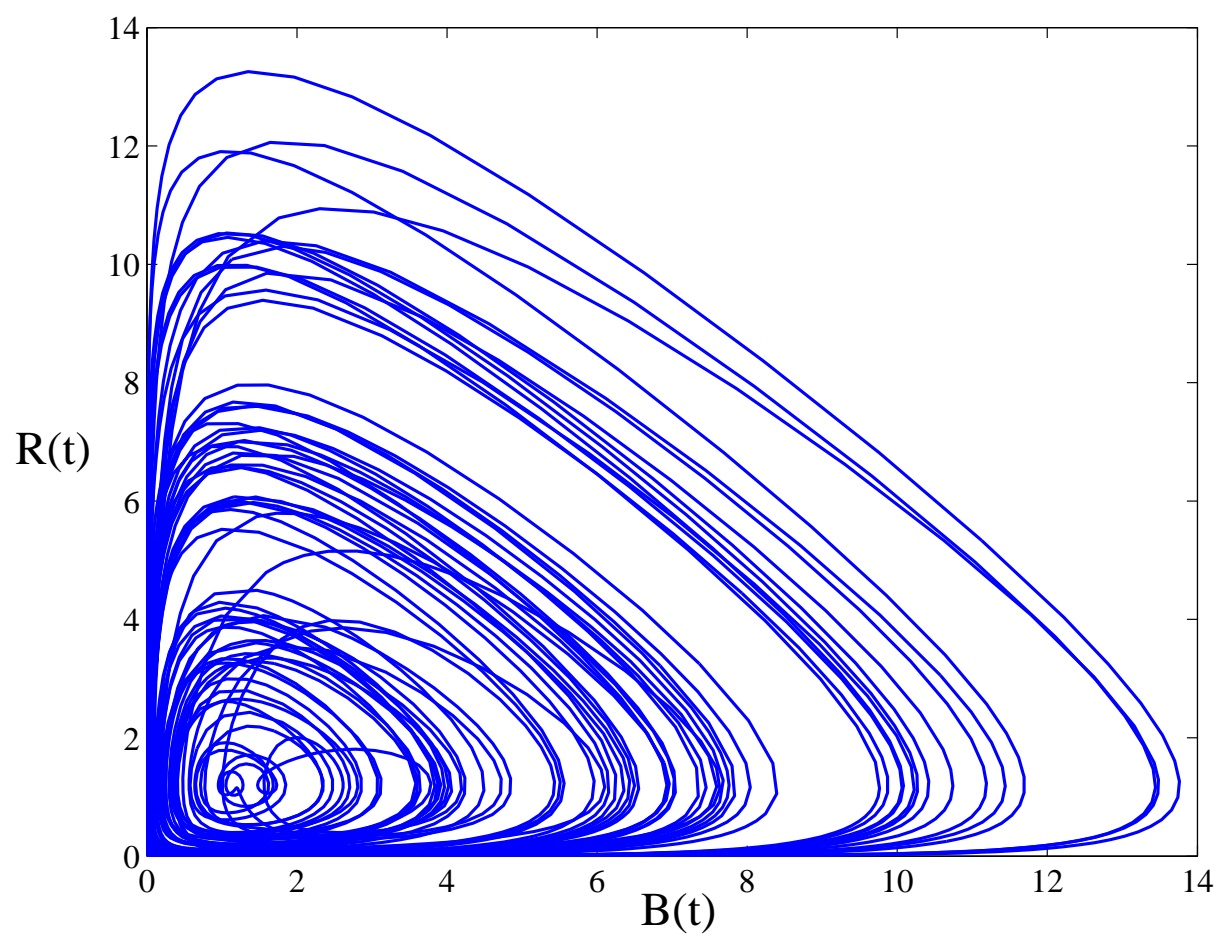

FiguRE 12: Phase orbits for $B(t)$ against $R(t)$, for forcing amplitude $\beta_{1}=0.2$ and frequency $\Omega=1.362$. 


\section{Discussion}

This article presents a detailed mathematical investigation of the structure of the solutions to the two level trophic food web model apparently first proposed by Stone [11]. Although we simplified the model by assuming that nutrient is in vast over supply, the system is nevertheless non-linear, due to the interaction between competing species. As a result, the unforced model predicts five steady states. Of these equilibria, only one gives long term survival of all four species. There is another equilibrium at the origin of the phase space, corresponding to total extinction. Four of the equilibrium points are unstable, and the one for which all populations survive is a centre.

Seasonal or daily forcing has also been studied for this model, based on a sinusoidal variation in the breeding rate for the bacteria. At small forcing amplitude there was reasonable agreement between the linear and non-linear solutions, as expected. The linearized solution provides considerable insight into a number of aspects of the global behaviour, including the identification of two forcing frequencies at which primary resonance occurs. Agreement between the numerical results and the linearized solution for small amplitude therefore serves as a valuable check on the reliability of the non-linear solution.

For the parameter values used here, taken from the article by Stone [11], primary resonance was found to occur at $\Omega \approx 1$, which corresponds to a forcing frequency $\omega$ of the order of 1 day $^{-1}$. These results are therefore appropriate to daily forcing, consistent with Edwards and Brindley [2].

As the forcing amplitude is increased, the agreement with linearized theory breaks down, as expected. The non-linear results exhibit rich diversity of complex behaviour, including high period orbits, sub-harmonic resonances, quasi-periodicity and chaos. The results strongly suggest that, as amplitude is increased for a fixed forcing frequency, quasi-periodicity may lead to chaos through a Ruelle-Takens-Newhouse bifurcation. 
It is appropriate to ask whether, in a real biological system, the forcing amplitudes could ever become sufficiently large for the more exotic nonlinear effects presented here to be observed in practice. From Stone's article [11], the unforced bacterial breeding rate, in dimensionless variables, has the value $\beta_{0}=1.2$, and the relative perturbation to this due to forcing is simply $\beta_{1} / \beta_{0}$, from equation (5). The quasi-periodic solutions illustrated in Figures 9 and 11 were obtained with forcing amplitude $\beta_{1}=0.05$, and this represents only a $4 \%$ variation to the unforced reproduction rate. Even the chaotic results of Figures 10 and 12, with $\beta_{1}=0.2$, represent only a $17 \%$ variation, and we therefore conclude that highly non-linear solution behaviour, including chaos, is a likely feature of such trophic web systems.

It has been assumed here that the nutrients $\mathrm{N}$ are in unlimited supply. When this is no longer true, even more rich dynamical behaviour may be possible. In particular, the four dimensional system studied here is essentially degenerate, in the sense that the unforced equations give rise to equilibrium points that are centres. When variations in nutrient concentration are also allowed, it is possible that these points may allow Hopf bifurcations to be present, so raising the additional complexity of limit cycles and their forced equivalents in the seasonally varying case. This is beyond the scope of this study, however, and is left for future work.

\section{References}

[1] L. Edelstein-Keshet. Mathematical Models in Biology. Random House, New York 1988. E26

[2] A. M. Edwards and J. Brindley. Zooplankton mortality and the dynamical behaviour of plankton population models. Bull. Math. Biol., 61:303-339, 1999. doi:10.1006/bulm.1998.0082 E25, E29, E52

[3] J. A. Freund, S. Mieruch, B. Scholze, K. Wiltshire and U. Feudel. Bloom dynamics in a seasonally forced phytoplankton-zooplankton 
model: Trigger mechanisms and timing effects. Ecological Complexity, 3:129-139, 2006. doi:10.1016/j.ecocom.2005.11.001 E25

[4] T. Gross, W. Ebenhöh and U. Feudel. Enrichment and foodchain stability: The impact of different forms of predator-prey interaction. $J$. Theor. Biol., 227:349-358, 2004. doi:10.1016/j.jtbi.2003.09.020 E26

[5] J. Guckenheimer and P. Holmes. Nonlinear Oscillations, Dynamical Systems and Bifurcations of Vector Fields. Springer-Verlag, New York 1983. E33, E34, E38

[6] A. Huppert, R. Olinky and L. Stone. Bottom-Up excitable models of phytoplankton blooms. Bull. Math. Biol., 66:865-878, 2004. doi:10.1016/j.bulm.2004.01.003 E25

[7] K. L. Kirk. Enrichment can stabilize population dynamics: Autotoxins and density dependence. Ecology, 79:2456-2462, 1998. doi:10.1890/0012-9658(1998)079[2456:ECSPDA]2.0.CO;2 E26

[8] L. Medio and M. Lines. Nonlinear dynamics a primer. Cambridge University Press, Cambridge 2001. E34

[9] J. D. Murray. Mathematical Biology. Springer-Verlag, New York 1989. E25, E26, E29, E31, E33

[10] J. C. Sprott, J. C. Wildenberg and Y. Azizi. A simple spatiotemporal chaotic Lotka-Volterra model. Chaos Solitons and Fractals, 26:1035-1043, 2005. doi:10.1016/j.chaos.2005.02.015 E49

[11] L. Stone. Phytoplankton-bacteria-protozoa interactions: a qualitative model portraying indirect effects. Mar. Ecol. Prog. Ser., 64:137-145, 1990. http://www. int-res. com/articles/meps/64/m064p137.pdf E25, E26, E27, E28, E34, E36, E52, E53

[12] J. M. T. Thompson and H. B. Stewart. Nonlinear Dynamics and Chaos. John Wiley and Sons, New York 1989. E49 
[13] J. E. Truscott and J. Brindley. Ocean plankton populations as excitable media. Bull. Math. Biol., 56:981-998, 1994. doi:10.1016/S0092-8240(05)80300-3 E27

\section{Author addresses}

1. S. A. Hadley, Department of Defence, Anglesea Barracks, Hobart, Tasmania 7000, Australia.

mailto:s_hadley@postoffice.utas.edu.au

2. L. K. Forbes, School of Maths and Physics, University of Tasmania, Private Bag 37, Hobart, Tasmania 7001, Australia. 